\title{
Estructura y fenología reproductiva de una población remanente de Adesmia bijuga Phil., Fabaceae, en un hábitat costero mediterráneo perturbado de Chile central
}

\section{Structure and breeding phenology of a remaining population of Adesmia bijuga Phil., Fabaceae, in a mediterranean coastal habitat disturbed of central Chile}

\author{
Persy Gómez ${ }^{1}$, Steffen Hahn ${ }^{1} \&$ José San Martín²* \\ 'Jardín Botánico, Universidad de Talca, Casilla 747, Talca, Chile. \\ 2Instituto de Biología Vegetal y Biotecnología, Universidad de Talca, Casilla 747, Talca, Chile. \\ *jsanmart@utalca.cl
}

\begin{abstract}
In this note we examine the population structure and breeding phenology of Adesmia bijuga Phil. The area of development of the study was Curepto, Maule Región, central Chile. Our results indicate a high vulnerability of local extinction of the studied population due low population size and disturbed habitat. Flowering and fructification of individuals exists, nevertheless absence of seedlings suggesting restrictions to the regeneration in the population of $A$. bijuga. It is recommended to continue with the research. Actions of conservation ex situ for the species are realized by the botanical garden of the Talca University.
\end{abstract}

Chile central es un área que alberga una gran diversidad de especies endémicas, por lo cual, a nivel mundial ha sido reconocida como una de las zonas prioritarias para conservar (Cowling et al. 1996, Myers et al. 2000, Gómez et al. 2012). Para el área costera entre los $35^{\circ} 50^{\prime}-36^{\circ} 50^{\prime} \mathrm{S}$ la vegetación nativa dominante es el Bosque Maulino costero con las especies endémicas Nothofagus glauca (Phil.) Krasser $y N$. alessandrii Espinosa (Donoso 1975), pero cuya superficie se ha reducido en un 67\%, dando paso a plantaciones de Pinus radiata D. Don (Echeverría et al. 2006). En la actualidad el paisaje se presenta fragmentado y degradado con pérdida de hábitat y en parches de vegetación nativa rodeados por las plantaciones (Bustamante \& Grez 1995, San Martín \& Donoso 1996, Bustamante \& Castor 1998, Bustamante et al. 2005, Gómez et al. 2012). Bajo este escenario se encuentra el arbusto Adesmia bijuga Phil., declarada especie en peligro crítico (Hahn \& Gómez 2008, Gómez et al. 2009, Gómez et al. 2012). Desde el punto de vista científico, la especie tiene importancia florística, biogeográfica y de conservación, a causa de su carácter endémico, hábitat vulnerable y restringida distribución espacial.

Las tres poblaciones de A. bijuga hasta ahora conocidas se encuentran en tres localidades costeras de la Región del Maule (Huelón, Curepto y Fundos San Pedro I y San Pedro II, Las Cañas, Constitución) (Gómez et al. 2009, Gómez \& Hahn 2010, Gómez et al. 2012) (Fig. 1). En la actualidad los sitios están bajo régimen de tenencia privada, e históricamente, sometidos a procesos de deforestación y cambio de uso de suelo, lo que ha alterado las condiciones del entorno natural. A. bijuga, supuestamente a causa de su escasa abundancia y uso, es desconocida en su biología y ecología y no se dispone de mayor información básica que entregue antecedentes de la recuperación natural de sus poblaciones (Hahn \& Gómez 2008, Gómez et al. 2009, Gómez \& Hahn 2010, Gómez et al. 2012).

Para contribuir a ampliar la información de historia natural de $A$. bijuga y dado que no existen estudios publicados donde se evalúen, en forma cuantitativa y experimental, procesos relativos a la regeneración, como la formación de flores, frutos o establecimiento de plántulas en esta especie, se realizó esta investigación que tuvo por objetivo describir las distintas fases fenológicas reproductivas de $A$. bijuga. El estudio poblacional y fenológico se desarrolló in situ en la Comuna de Curepto, Provincia de Talca, Región del Maule y realizando un conteo de todos los individuos vivos agrupándolos según tamaño en: adultos, jóvenes y plántulas. Aunque entran en la contabilidad un segundo grupo como muertos con ejes en pie, ausencia de hojas y yemas, y con aspecto seco y quebradizo. Como mediciones se evaluó la altura de tamaño $(\mathrm{cm})$ de cada individuo vivo y luego la fenología siguiendo a Henríquez et al. (2012) en sólo 10 individuos marcados. La selección se condicionó a la 
existencia de ejemplares adultos dentro del área de estudio con evidencias reproductivas pasadas y que garantizaran nuevas floraciones y fructificaciones, no así los ejemplares jóvenes, que se descartaron a causa del riesgo de alcanzar las fenofases señaladas. Como el objetivo era describir las fenofases reproductivas y no el crecimiento y la persistencia foliar, el monitoreo se realizó durante seis meses (noviembre 2012 a abril de 2013). En el estudio se evaluó cada una de las siguientes fenofases: botones florales-preantesis, flores o antesis y frutos, para luego construir un diagrama de fenofases, de acuerdo a Castro-Diez \& Monserrat-Martí (1998), Milla et al. (2010) y Henríquez et al. (2012). Finalmente para cada fenofase se estimó la proporción de individuos de acuerdo a las siguientes categorías: Fenofase

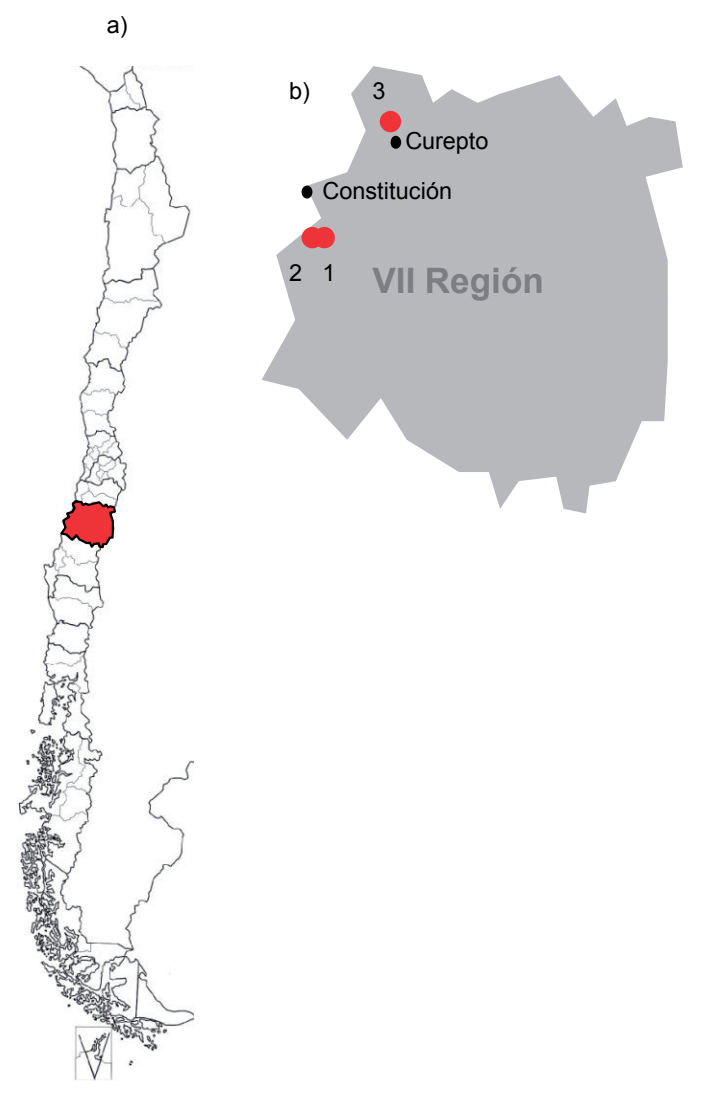

FIgURA 1. Ubicación geográfica de las poblaciones donde se encuentra Adesmia bijuga. (a) Mapa de Chile caracterizando la VII Región del Maule. (b) Acercamiento de la VII Región del Maule con las tres únicas localidades en que se ha encontrado $A$. bijuga, 1) Población del Fundo San Pedro I, Las Cañas, Constitución, 2) Población Fundo San Pedro II, Las Cañas, Constitución y 3) Población de Huelón, Curepto.

FIGURE 1. Geographic location of Adesmia bijuga populations. (a) Map of Chile characterizing the Maule Region. (b) Close-up of the Maule Region with three locations of A. bijuga, 1) Population Fundo San Pedro I, Las Cañas, Constitucion, 2) Population Fundo San Pedro II, Las Cañas, Constitucion and 3) Population Huelón, Curepto.
I: en más de $25 \%$ de las plantas, Fenofase II: en 6 a $25 \%$, Fenofase III: $5 \%$ o menos.

La población total evaluada fue de 363 individuos de los cuales sólo $25(6,9 \%)$ se encontraban vivos y localizados en un sitio y aspecto agrupado bajo dosel de una plantación de Pinus radiata con individuos distribuidos en claros y sectores de menor cobertura arbórea y en márgenes de un camino forestal. Durante el monitoreo se realizó un recorrido, revisión e inspección ocular en el piso más allá de los individuos marcados sin hallazgos de plántulas y juveniles, lo cual sugiere que la regeneración de la especie está en declinación (Fig. 2). Este resultado para Curepto (Región del Maule) sugiere que la especie enfrenta dificultades de reclutamiento, regeneración y de sobrevivencia (93,1\% de individuos muertos), por lo cual se hace necesario mantener un monitoreo, como también determinar si el factor que limita la actividad reproductiva tiene origen en la planta misma (internas) o ambientales (externas). Al mismo tiempo surgen nuevas interrogantes relacionadas con el modelo reproductivo de la especie y que afecta la forma de evaluar su biología poblacional. $\mathrm{Si}$ es favorecida la reproducción clonal, es esperable que entorno a la planta madre se desarrollen individuos de tamaños diferentes y que dada su conectividad tendrían una fisionomía similar. Este estudio no es el caso, ya que en la contabilidad se consideraron sólo individuos unitarios. La existencia de sólo un $6,9 \%$ de individuos vivos sugiere nuevas hipótesis de trabajo relacionadas con la ecología y fisiología de la especie así como antecedentes históricos de los procesos de disturbio del sitio.

En cuanto a la fenología reproductiva, los primeros botones florales y flores se desarrollaron en noviembre, y los primeros frutos a partir de diciembre. En las fenofases se observó cierta sincronía con expresión de todas las fases de botones florales, flores y frutos. La floración es sostenida puesto que más del $25 \%$ de la población de A. bijuga presentó flores 4 meses (Fig. 3). Un 40\% de los individuos formaron flores y frutos. Los resultados sugieren que las restricciones a la regeneración en la población de $A$. bijuga estudiada podrían estar vinculadas a la herbivoría floral (Gómez et al. 2012), depredación de semillas predispersión (Hahn \& Gómez 2008), o a procesos postdispersión como germinación de semillas y establecimiento de plántulas (Henríquez et al. 2012).

Sin duda que en Curepto el estado de $A$. bijuga responde a una etapa de la dinámica poblacional como efectos de un proceso histórico y problemas en un ambiente perturbado. Asímismo es desconocido el tamaño original de la población y el impacto de los procesos que implica la sustitución de la vegetación original. La población actual remanente demuestra resistencia y, aparentemente, una débil capacidad adaptativa al alcanzar los individuos a reproducirse aunque de modo deficiente.

Se concluye que la población de $A$. bijuga en Curepto, 
presenta una alta vulnerabilidad de extinción local, debido principalmente a su pequeño tamaño poblacional y baja tasa reproductiva. Además, de estar inmersa en un ambiente alterado dominado por plantaciones de $P$. radiata. Por otra parte, poblaciones pequeñas tienen una mayor probabilidad de extinguirse debido a pérdida de variabilidad genética, variación demográfica y variación ambiental (Gilpin \& Soulé 1986).

Se sugiere continuar y diversificar otros estudios en esta especie con problemas de sobrevivencia in situ. Por otro lado, se hace necesario profundizar la ecología de la demografía poblacional e investigar si la estrategia reproductiva es sobrepasada por barreras ambientales o de la especie misma cuando entra a un proceso regresivo. Afortunadamente, en condiciones artificiales la regeneración vegetativa de $A$. bijuga responde adecuadamente como también su crecimiento ex situ bajo sol directo según lo informado en ensayos realizados en el Jardín Botánico de la Universidad de Talca.

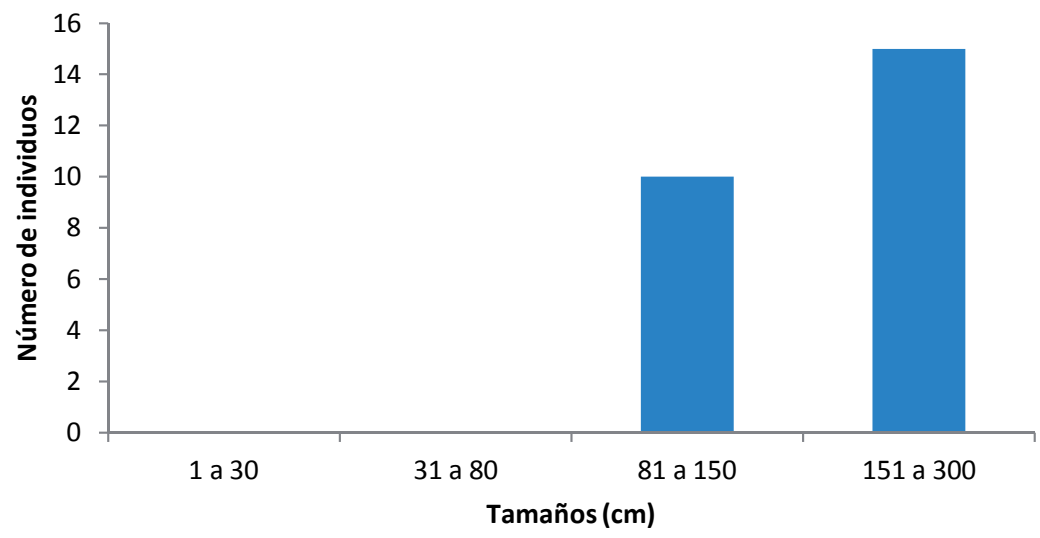

FIgURA 2. Estructura de tamaños para una población de A. bijuga (individuos vivos), Huelón, Curepto, Región del Maule, Chile central.

Figure 2. Sizes structure for A. bijuga population (living individuals), Huelón, Curepto, Maule Region, central Chile.

\section{Nov Dic Ener Febr Mar Abr}

\section{Botón Floral}

Flores

\section{Frutos}

FiguRa 3. Diagrama de fenofases reproductivas de A. bijuga. Los niveles de frecuencia en la población están indicados por las líneas, doble línea (fenofase I $>25 \%$ de la población), línea simple (fenofase II entre 5-25\% de la población), línea punteada (fenofase III $<5 \%$ de la población).

Figure 3. Phenophase reproductive diagram of A. bijuga. The levels of frecuency in the populations are indicated by doble line (phenophase I $>25 \%$ of the population), simple line (phenophase II 5-25\% of the population), and broken line (phenophase III $<5 \%$ of the population).

\section{AGRADECIMIENTOS}

Los autores agradecen el apoyo de la Dirección de Investigación (DI), Jardín Botánico e Instituto de Biología de la Universidad de Talca, Región del Maule, Chile, así como a revisores anónimos que por sus comentarios han contribuido a mejorar el trabajo.

\section{BIBLIOGRAFÍA}

Bustamante, R. \& A. Grez. 1995. Consecuencias ecológicas de la fragmentación de los bosques nativos. Ambiente y Desarrollo 11: 58-63.

Bustamante, R. \& C. Castor. 1998 The decline of an endangered temperate ecosystem: the ruil (Nothofagus alessandrii) forest in central Chile. Biodiversity and Conservation 7: 
1607-1626.

Bustamante, R., J. Simonetti, A. Grez \& J. San Martín. 2005. Fragmentación y dinámica de regeneración del bosque maulino: diagnóstico actual y perspectivas futuras. En: C. Smith-Ramírez, J. Armesto \& C. Valdovinos (eds.). Historia, biodiversidad y ecología de los bosques costeros de Chile. 555-564. Editorial Universitaria, Santiago.

Castro-Díez, P. \& G. Monserrat-Martí. 1998. Phenological pattern of fifteen Mediterranean phanaerophytes from Quercus ilex communities of NE-Spain. Plant Ecology 139: 103-112.

Cowling, L., PH. Rundel, B. Lamont, M.K. Arroyo \& M. ARIANOUTSOU. 1996. Plant diversity in mediterraneanclimate regions. Trend in Ecology \& Evolution 11(9): $362-$ 366.

Donoso, C. 1975. Distribución ecológica de la especies de Nothofagus en la zona mesomórfica. Universidad de Chile, Facultad de Ciencias Forestales, Boletín Técnico 33: 1-21.

Echeverría, R., A. Newton, A. Lara, JM. Rey-Benayas \& D. CoOmes. 2006. Impacts of forest fragmentation on species composition and forest structure in the temperate landscape of southern Chile. Global Ecology and Biogeography 16: 426-439.

Gilpin, M.E. \& M.E. SoulÉ. 1986. Minimum viable populations: processes of species extinction. In: M.E. Soulé (ed.), Conservation Biology: The Science of Scarcity and Diversity. Sinauer, Sunderland, MA, pp. 19-34.

Gómez, P., S. Hahn \& J. SAn Martín. 2009. Estructura y composición florística de un matorral bajo plantaciones de Pinus radiata D. Don en Chile Central. Gayana Botánica 66(2): 252-264.

Gómez, P. \& S. Hahn. 2010. Nueva localidad para Adesmia bijuga Phil. (Fabaceae), un endemismo de la Región del Maule (VII), Chile. Chloris chilensis año 13. N². URL: http:/ www.chlorischile.cl

Gómez, P., D. Lillo \& AV. GonzÁlez. 2012. Polinización y sistema reproductivo en Adesmia bijuga Phil. (Fabaceae), una especie en peligro crítico en Chile central. Gayana Botánica 69(2): 286-295.

Hahn, S. \& P. Gómez. 2008. Hallazgo de Adesmia bijuga Phil. (Fabaceae) en la zona costera, Región del Maule, Chile central. Gayana Botánica 65(1): 119-121.

Henríquez, CA., G. Sotes \& R. Bustamante. 2012. Fenología reproductiva de Pouteria splendens (Sapotaceae). Gayana Botánica 69(2): 251-255.

Milla, R., P. Castro-Diez \& G. Monserrat-Martí. 2010. Phenology of Mediterranean woody plants from NE Spain: synchrony, seasonality, and relationships among phenophases. Flora 205: 190-199.

Myers, N., R.A. Mittermeier, C.G. Mittermeier, G.A.B. DA FonsecA \& J. Kent. 2000. Biodiversity hotspots for conservation priorities. Nature 403: 853-858.

SAn Martín, J. \& C. Donoso. 1996. Estructura e impacto antrópico en el bosque maulino de Chile. En: JJ. Armesto, C. Villagrán \& M.T. K. Arroyo (eds.), Ecología de los bosques nativos de Chile. 153-168. Editorial Universitaria, Santiago.

Recibido: 10.07 .13

Aceptado: 11.10 .13 